PROCEEDINGS OF THE

AMERICAN MATHEMATICAL SOCIETY

Volume 140, Number 7, July 2012, Pages 2417-2427

S 0002-9939(2011)11252-8

Article electronically published on November 15, 2011

\title{
GERM HYPOELLIPTICITY AND LOSS OF DERIVATIVES
}

\author{
GREGORIO CHINNI \\ (Communicated by Franc Forstneric) \\ AbStRACt. We prove hypoellipticity in the sense of germs for the operator

$$
\mathcal{P}=L_{q} \bar{L}_{q}+\bar{L}_{q} t^{2 k} L_{q}+Q^{2},
$$ \\ where

$$
L_{q}=D_{t}+i t^{q-1} \sqrt{-\Delta_{x}} \text { and } Q=x_{1} D_{2}-x_{2} D_{1},
$$ \\ even though it fails to be hypoelliptic in the strong sense. The primary tool is \\ an a priori estimate.
}

\section{INTRODUCTION}

In 2005 J. J. Kohn published a seminal paper, 7], devoted to the problem of $C^{\infty}$-hypoellipticity of operators being sums of squares of complex vector fields. In particular he proposed and studied a model operator of the form

$$
P_{k}=L \bar{L}+\bar{L}|z|^{2 k} L \quad \text { with } \quad L=\frac{\partial}{\partial z}+i \bar{z} \frac{\partial}{\partial t} .
$$

Later on A. Bove, M. Derridj, J. J. Kohn, and D. S. Tartakoff, 2], considered a generalization of that operator and proved both the $C^{\infty}$-hypoellipticity and the $C^{\omega}$-hypoellipticity, i.e., its analytic hypoellipticity.

One of the points of Kohn's paper is that, in contrast with the case of real vector fields satisfying the Hörmander bracket condition, a sum of squares of complex vector fields can be hypoelliptic and, at the same time, lose an arbitrary number of derivatives. As a consequence the primary tool to show its hypoellipticity, the localized a priori estimate, can be rather difficult to obtain.

M. Christ, [5], has shown that one may add a variable and consider the operator $P_{k}+D_{y}^{2}, y$ being the added real variable, and obtain an operator which is not hypoelliptic, provided $k>0$, i.e., when $P_{k}$ is not subelliptic. Thus we have an example, even though in three variables, of an operator being the sum of squares of complex vector fields, satisfying the complex Hörmander bracket condition for which hypoellipticity fails.

On the other hand, in the subelliptic case, i.e., real vector fields satisfying the Hörmander bracket condition, the problem of analytic hypoellipticity - of course when the vector fields have real analytic coefficients - is still open, and it is well known that there are both $C^{\infty_{-}}, C^{\omega}$-hypoelliptic and $C^{\infty}$ - but not $C^{\omega}$-hypoelliptic operators. An attempt to understand this situation is Treves conjecture. We refer to the cited literature for details.

Received by the editors February 23, 2011.

2010 Mathematics Subject Classification. Primary 35H10, 35A27.

(C)2011 American Mathematical Society Reverts to public domain 28 years from publication 
Hanges, 6], and Bove, Derridj, Tartakoff, 3], have shown that, for sums of squares of real vector fields, i.e. subelliptic operators, if one focuses on germ analytic hypoellipticity rather than on hypoellipticity, there are operators which are germ analytic hypoelliptic even though they are not analytic hypoelliptic. In [3] a connection is established between this fact and the microlocal Treves conjecture, in particular asserting that there is no contradiction between the conjecture and this phenomenon.

For sums of squares of complex vector fields the scenario is more involved (see, e.g., 4.) In particular $C^{\infty}$-hypoellipticity is not guaranteed anymore by the bracket condition.

The purpose of this paper is to study the germ $C^{\infty}$-hypoellipticity for the operator

$$
\mathcal{P}=L_{q} \bar{L}_{q}+\bar{L}_{q} t^{2 k} L_{q}+Q^{2}
$$

where $L_{q}=D_{t}+i t^{q-1} \sqrt{-\Delta_{x}}, q$ is an even integer, $Q=x_{1} D_{2}-x_{2} D_{1}$, and $k \in \mathbb{N}$. Here $\sqrt{-\Delta_{x}}$ denotes the pseudodifferential operator which, in Fourier variables, multiplies by $\|\xi\|$ the Fourier transform of a distribution.

We prove

Theorem 1.1. The operator $\mathcal{P}$ is $C^{\infty}$-hypoelliptic in the sense of germs on circular cilinders centered at the origin in the $x$ variables or, more generally, on regions with azimuthal symmetry.

The result is obtained following the ideas in [2].

We want to add just a few brief remarks.

First the condition that $q$ is even is no loss of generality, since the operator $\mathcal{P}$ with $q$ odd is subelliptic and hence hypoelliptic.

The operator in (1.1) is not exactly a sum of squares of complex vector fields since it involves a pseudodifferential operator. We are not aware of a differential example of the situation we present here.

Even though the case of the operator $\mathcal{P}$ is not contained in Christ's paper [5], one may repeat almost verbatim that proof, e.g., with $q=2$, and show that $\mathcal{P}$ is not $C^{\infty}$-hypoelliptic. Thus in shifting from $C^{\infty}$-hypoellipticity to $C^{\infty}$-hypoellipticity in the sense of germs there is some gain.

\section{Hypoellipticity IN THE SENSE OF GERMS}

Preliminary remarks and notation. Christ in [5] has shown that Kohn's operator plus the square of a derivative in an added variable is not hypoelliptic. Arguing analogously we may show that $\mathcal{P}$, at least for certain values of the parameters, is not hypoelliptic.

We recall that a differential operator $P$, defined in $\Omega$, an open set of $\mathbb{R}^{n}$, is said to be germ hypoelliptic at a compact set $K \subset \Omega$ if for each open set $U \supset K$ there is an open set $V$ with $U \supset V \supset K$ such that for all $u \in \mathcal{D}^{\prime}(U), P u \in C^{\infty}(U) \Rightarrow$ $u \in C^{\infty}(V)$. In particular $P$ is germ hypoelliptic at a point $x_{0} \in \Omega$ if $P$ is germ hypoelliptic at $K=\left\{x_{0}\right\}$. In our case we choose $K$ equal to $\{(t=0, x=0)\}$ or $\{t=0,|x| \leqslant c\}$.

We use the same notation as in [2]: R.J., meaning relative junk, shall denote any multiple of terms in the left-hand side of an estimate (see below, e.g., (2.9)) but with lower Sobolev index and possibly a radial derivative on the localizing function. Besides that, by R.J. we also mean those multiples of terms in the left-hand side of 
(2.9) again with lower Sobolev index but in which a new localizing radial function equal to 1 on the support of the original localizing function $\varphi$ has appeared. The relative junk terms are terms which can be iteratively estimated in the end.

Another technical observation concerns the localizing function used in proving the a priori estimate, $\varphi$ : it will be a radial function of the $x$-variables only, with small support and equal to 1 near the origin. Its radial nature is closely related to the presence of the vector field $Q$ : the projection of the bicharacteristic curves of $Q$ on the space of variables comprises the level curves of the radial localizing function $\varphi$. The cut-off functions we use are smooth compactly supported functions of the $x$ variables only. This is allowed since any dependence on the $t$-variable would imply that any derivative of the localizing function localizes the operator in an elliptic region, where the hypoellipticity result is granted. We emphasize that the radial nature of the localizing function is a necessary condition to obtain the localization estimate (a key step to obtain the result). We remark in addition that since

$$
\sigma\left(\left[L_{q}, \varphi\right]\right)=i t^{q-1} \sum_{k \geq 1}\left(\sum_{|\alpha|=k} D_{x}^{\alpha} \varphi \partial_{\xi}^{\alpha}(\|\xi\|) / \alpha !\right)
$$

and $\sum_{|\alpha|=k} D_{x}^{\alpha} \varphi \partial_{\xi}^{\alpha}(\|\xi\|) \in S_{1,0}^{1-|\alpha|}$, then there exist $a_{0} \in S_{1,0}^{0}$, unique modulo $S^{-\infty}$, such that, for any $N \in \mathbb{N}, a_{0}(x, \xi)-\sum_{1 \leq k<N}\left(\sum_{|\alpha|=k} D_{x}^{\alpha} \varphi \partial_{\xi}^{\alpha}(\|\xi\|) / \alpha !\right) \in$ $S_{1,0}^{-N}$. Equivalently, there exist $b_{0} \in S_{1,0}^{0}$ and $b_{-1} \in S_{1,0}^{-1}$ such that $\sigma\left(\left[L_{q}, \varphi\right]\right) \sim$ $t^{q-1}\left(\varphi^{(1)} b_{0}+b_{-1}\right)$ modulo $S^{-\infty}$. Here $\varphi^{(1)}=\partial_{r} \varphi$ denotes the radial derivative of $\varphi$.

The characteristic set of $\mathcal{P}$,

$\operatorname{Char}(\mathcal{P})=\left\{(t, x, \tau, \xi) \in T^{*} \mathbb{R}^{3} \backslash 0: t=0=\tau, x_{1} \xi_{2}-x_{2} \xi_{1}=0\right.$ with $\left.\xi_{1}^{2}+\xi_{2}^{2} \neq 0\right\}$,

is not symplectic, but the bicharacteristic curves of $\mathcal{P}$, i.e., the Hamilton leaves of the foliation of Char $(\mathcal{P})$, are closed curves foliating a suitable neighborhood of a characteristic base point on which the hypoellipticity in the sense of germs is going to be obtained. Since our result is essentially microlocal near $\tau=0$, any Sobolev norm can be replaced by a Sobolev norm in the $x$-variables, $L_{t}^{2} \times H_{x}^{s}$, that is, the Sobolev norms defined by $\|u\|_{s}^{2}=\left\|\Lambda^{s} u\right\|_{0}^{2}$ where $\Lambda^{s}$ denotes the pseudo-differential operator with symbol $\left(1+\|\xi\|^{2}\right)^{\frac{s}{2}}$. An important property of $\Lambda^{s}$ is that it commutes with $\mathcal{P}$.

We state now, without proof, some useful estimates. Let $u \in C_{0}^{\infty}(\Omega)$. We have

$$
\left\|t^{r} u\right\|_{s}^{2} \leq \text { s.c. }\left\|t^{r-a} u\right\|_{s-\frac{a}{q}}^{2}+\text { l.c. }\left\|t^{r+b} u\right\|_{s+\frac{b}{q}}^{2}, \quad a \leq r,
$$

where the notation "s.c." and "l.c." denotes an "arbitrary small positive constant" and a "large constant". The same estimate can be obtained exchanging the roles of s.c. and l.c.:

$$
\left\|t^{r} u\right\|_{s}^{2} \leq \text { l.c. }\left\|t^{r-a} u\right\|_{s-\frac{a}{q}}^{2}+\text { s.c. }\left\|t^{r+b} u\right\|_{s+\frac{b}{q}}^{2}, \quad a \leq r .
$$


Keep in mind that $\left[\bar{L}_{q}, L_{q}\right]=2(q-1) t^{q-2} \sqrt{-\Delta_{x}}$ and $L_{q}-\bar{L}_{q}=2 i t^{q-1} \sqrt{-\Delta_{x}}$. We also have

$$
\begin{aligned}
& \left\|\bar{L}_{q} u\right\|_{s}^{2}+\left\|t^{\frac{q-2}{2}} u\right\|_{\frac{1}{2}+s}^{2} \leq\left\|L_{q} u\right\|_{s}^{2}+\|u\|_{s}^{2} ; \\
& \left\|t^{\frac{q-2}{2}} u\right\|_{s+\frac{1}{2}}^{2} \leq\left\|L_{q} u\right\|_{s}^{2}+\left\|\bar{L}_{q} u\right\|_{s}^{2} ; \\
& \left\|t^{q-1} u\right\|_{s}^{2} \leq\left\|L_{q} u\right\|_{s-1}^{2}+\left\|\bar{L}_{q} u\right\|_{s-1}^{2}+\left\|t^{q-1} u\right\|_{s-1}^{2} .
\end{aligned}
$$

We also point out that the same result can be obtained on corona shaped sets, i.e., sets of the type $K=\left\{\left(0, x_{1}, x_{2}\right): c_{1} \leq x_{1}^{2}+x_{2}^{2} \leq c^{2}\right.$ with $\left.c_{2} \geq c_{1} \geq 0\right\}$. Sure enough, the localizing function $\varphi$ must again be chosen radial and equal 1 near $K$.

First we give a short proof of a generalization of a classical a priori estimate for $\mathcal{P}$.

Lemma 2.1. For $u \in C_{0}^{\infty}(\Omega), \Omega$ an open subset of $\mathbb{R}^{n}$, we have

$$
\|u\|_{-\frac{k-1}{q}}^{2}+\left\|\bar{L}_{q} u\right\|_{0}^{2}+\left\|t^{k} L_{q} u\right\|_{0}^{2}+\|Q u\|_{0}^{2} \leq C\langle\mathcal{P} u, u\rangle .
$$

Let $r \in \mathbb{R}$. We have

$$
\begin{aligned}
\|u\|_{r}^{2}=\left|\left(\left[L_{q}, i t\right] \Lambda^{r} u, \Lambda^{r} u\right)\right| & \leq\left|\left(L_{q} t \Lambda^{r} u, \Lambda^{r} u\right)\right|+\left|\left(t L_{q} \Lambda^{r} u, \Lambda^{r} u\right)\right| \\
& =\left|\left(t \Lambda^{r} u, \bar{L}_{q} \Lambda^{r} u\right)\right|+\left|\left(L_{q} \Lambda^{r-\epsilon} u, t \Lambda^{r+\epsilon} u\right)\right| .
\end{aligned}
$$

Now

$$
\left|\left(L_{q} \Lambda^{r-\epsilon} u, t \Lambda^{r+\epsilon} u\right)\right| \leq s . c .\left\|L_{q} \Lambda^{r-\epsilon} u\right\|_{0}^{2}+\text { l.c. }\left\|t \Lambda^{r+\epsilon} u\right\|_{0}^{2} ;
$$

the last term can thus be estimated:

$$
\begin{aligned}
\left\|t \Lambda^{r+\epsilon} u\right\|_{0}^{2}=\left(t^{2} \Lambda^{r-2 \epsilon} u\right. & \left., \Lambda^{r} u\right) \leq l . c .\left\|t^{2} \Lambda^{r-2 \epsilon} u\right\|_{0}^{2}+\text { s.c. }\left\|\Lambda^{r} u\right\|_{0}^{2} \\
& \leq \ldots \leq l . c .\left\|t^{\gamma} \Lambda^{r+\gamma \epsilon} u\right\|_{0}^{2}+\text { s.c. }\left\|\Lambda^{r} u\right\|_{0}^{2} .
\end{aligned}
$$

The first term in the right-hand side of (2.6) can be estimated as

$$
\begin{aligned}
\left\|L_{q} \Lambda^{r-\epsilon} u\right\|_{0}^{2} & =\left(L_{q} \Lambda^{r-\epsilon} u, L_{q} \Lambda^{r-\epsilon} u\right)=\left(\bar{L}_{q} L_{q} \Lambda^{r-\epsilon} u, \Lambda^{r-\epsilon} u\right) \\
& =\left(L_{q} \bar{L}_{q} \Lambda^{r-\epsilon} u, \Lambda^{r-\epsilon} u\right)+\left(\left[\bar{L}_{q}, L_{q}\right] \Lambda^{r-\epsilon} u, \Lambda^{r-\epsilon} u\right) \\
& =\left\|\bar{L}_{q} \Lambda^{r-\epsilon} u\right\|_{0}^{2}+\left\|t^{\frac{q-2}{2}} \Lambda^{r-\epsilon+\frac{1}{2}} u\right\|_{0}^{2} \\
& \leq \ldots \leq\left\|\bar{L}_{q} \Lambda^{r-\epsilon} u\right\|_{0}^{2}+\text { l.c. }\left\|t^{s(q-2)} \Lambda^{r+s(1-2 \epsilon)} u\right\|_{0}^{2}+\text { s.c. }\left\|\Lambda^{r} u\right\|_{0}^{2} .
\end{aligned}
$$

Keeping in mind that $\left[L_{q}, t^{k}\right]=k t^{k-1}$ and imposing the conditions $2 s(q-2)=k-1$, $2 \gamma=k-1, r+2 \gamma \epsilon=0$ and $r+2 s(1-2 \epsilon)=0$, we obtain that $\epsilon=\frac{1}{q}$ and $r=-\frac{(k-1)}{q}$. Thus

$$
\|u\|_{-\frac{k-1}{q}}^{2}+\left\|\bar{L}_{q} u\right\|_{0}^{2}+\left\|t^{k} L_{q} u\right\|_{0}^{2}+\|Q u\|_{0}^{2} \leq C\langle\mathcal{P} u, u\rangle .
$$

Localization of the estimate. Let us first consider the case $k=1$. Let $\varphi$ be as above and let $u \in C^{\infty}(\Omega)$ with small support in the $t$-variable. We have

$$
\begin{aligned}
\|\varphi u\|_{0}^{2} & =\left|\left(\left[L_{q}, i t\right] \varphi u, \varphi u\right)\right| \leqslant\left|\left(t L_{q} \varphi u, \varphi u\right)\right|+\left|\left(L_{q} t \varphi u, \varphi u\right)\right| \\
& \leqslant \underbrace{\mid\left(t \varphi L_{q} u, \varphi u\right)}_{I} \mid+\underbrace{\left|\left(t \varphi u, \varphi \bar{L}_{q} u\right)\right|}_{I I}+\underbrace{\left|\left(t\left[L_{q}, \varphi\right] u, \varphi u\right)\right|}_{I I I}+\underbrace{\left|\left(t \varphi u,\left[\bar{L}_{q}, \varphi\right] u\right)\right|}_{I V} .
\end{aligned}
$$

We study each single term:

$$
(I) \leqslant l . c .\left\|\varphi t L_{q} u\right\|_{0}^{2}+\text { s.c. }\|\varphi u\|_{0}^{2} \text { and }(I I) \leqslant l . c .\left\|\varphi \bar{L}_{q} u\right\|_{0}^{2}+\text { s.c. }\|\varphi u\|_{0}^{2} ;
$$


in view of the above argument

$$
(I I I),(I V) \leqslant l . c .\left\|t^{q} \varphi^{(1)} u\right\|_{0}^{2}+\text { s.c. }\|\varphi u\|_{0}^{2}+\text { const. }\|\tilde{\varphi} u\|_{-1}^{2}+R . J .,
$$

where $\tilde{\varphi}$ is again a radial cut-off function such that $\tilde{\varphi} \equiv 1$ on the support of $\varphi$. The term $\|\tilde{\varphi} u\|_{-1}^{2}$ is also relative junk. Here as well as in the sequel we absorb as relative junk those terms of the form $\|u\|_{-\infty}^{2}$, due to the action of regularizing operators. We have

$$
\|\varphi u\|_{0}^{2} \leqslant\left\|\varphi t L_{q} u\right\|_{0}^{2}+\left\|\varphi \bar{L}_{q} u\right\|_{0}^{2}+\|\varphi Q u\|_{0}^{2}+\left\|t^{q} \varphi^{(1)} u\right\|_{0}^{2}+R . J .
$$

The term $\left\|t^{q} \varphi^{(1)} u\right\|_{0}^{2}$ is relative junk, since for any radial cut-off function $\phi$

$$
\left\|t^{\frac{q}{2}} \phi u\right\|_{0}^{2} \lesssim\|\phi u\|_{-\frac{1}{2}}+\|t \phi u\|_{-\frac{1}{2}}+\left\|t^{q} \phi_{1} u\right\|_{-\frac{1}{2}}+\left\|t \phi L_{q} u\right\|_{-\frac{1}{2}}
$$

where $\phi_{1}$ is radial and $\phi_{1} \equiv 1$ on the support of $\phi$.

On the other hand we have

$$
\begin{aligned}
\left\|t \varphi L_{q} u\right\|_{0}^{2}+ & \left\|\varphi \bar{L}_{q} u\right\|_{0}^{2}+\|\varphi Q u\|_{0}^{2} \\
\leq & |(\varphi \mathcal{P} u, \varphi u)|+\left|\left(t \varphi L_{q} u,\left[t \varphi, L_{q}\right] u\right)\right|+\left|\left(\varphi \bar{L}_{q} u,\left[\varphi, \bar{L}_{q}\right] u\right)\right| \\
\lesssim & \|\varphi \mathcal{P} u\|_{0}^{2}+\text { s.c. }\left(\|\varphi u\|_{0}^{2}+\left\|\varphi \bar{L}_{q} u\right\|_{0}^{2}+\left\|t \varphi L_{q} u\right\|_{0}^{2}\right) \\
& +\left\|t^{q-1} \varphi^{(1)} u\right\|_{0}^{2}+\left\|\tilde{\varphi} \bar{L}_{q} u\right\|_{-\frac{1}{2}}^{2}+\left\|t \tilde{\varphi} L_{q} u\right\|_{-\frac{1}{2}}^{2}+\|\tilde{\varphi} u\|_{-1}^{2}+\text { R.J. } \\
= & \|\varphi \mathcal{P} u\|_{0}^{2}+\text { s.c. }\left(\|\varphi u\|_{0}^{2}+\left\|\varphi \bar{L}_{q} u\right\|_{0}^{2}+\left\|t \varphi L_{q} u\right\|_{0}^{2}\right)+\text { R.J. }
\end{aligned}
$$

Hence we have

$$
\|\varphi u\|_{0}^{2}+\left\|t \varphi L_{q} u\right\|_{0}^{2}+\left\|\varphi \bar{L}_{q} u\right\|_{0}^{2}+\|\varphi Q u\|_{0}^{2} \lesssim\|\varphi \mathcal{P} u\|_{0}^{2}+R . J .
$$

Using the previous techniques, we can push down arbitrarily the Sobolev index of the R.J. terms and obtain

$$
\|\varphi u\|_{0}^{2} \lesssim\|\tilde{\varphi} \mathcal{P} u\|_{0}^{2}+\|\tilde{\varphi} u\|_{-N}^{2},
$$

where $\tilde{\varphi}$ is a radial cut-off function equal to 1 near the support of $\varphi$.

More generally the above estimate can be obtained with a general Sobolev index $s$. Let $\ell$ be such that $s-\ell \leq 0$. We think of $\sigma([L, \varphi])$ as a symbol of the form $\sigma([L, \varphi]) \sim t^{q-1}\left(\sum_{j=0}^{\ell-1} \varphi^{j+1} b_{-j}+b_{-\ell}\right)$, modulo $S^{-\infty}$, where $b_{-j} \in S_{1,0}^{-j}, b_{-\ell} \in S_{1,0}^{-\ell}$ and $\varphi^{(j+1)}=\partial_{r}^{j+1} \varphi$. In the case $s=0$ we have set $\sigma([L, \varphi]) \sim t^{q-1}\left(\varphi^{1} b_{0}+b_{-1}\right)$. Now again using the previous techniques to push down the Sobolev indexes, we obtain

$$
\|\varphi u\|_{s}^{2} \lesssim\left\|\varphi_{1} \mathcal{P} u\right\|_{s}^{2}+\left\|\varphi_{1} u\right\|_{-N}^{2}
$$

where $\varphi_{1}$ is a radial cut-off function equal to 1 on the support of $\varphi$.

Case $k>1$.

We want to establish an estimate of the type

$$
\begin{gathered}
c_{0}\|\varphi u\|_{0}^{2}+c_{1}\left\|\varphi \bar{L}_{q} u\right\|_{\frac{k-1}{q}}^{2}+c_{2}\left\|\varphi t^{k} L_{q} u\right\|_{\frac{k-1}{q}}^{2}+c_{3}\|\varphi Q u\|_{\frac{k-1}{q}}^{2}+c_{4}\left\|t^{\frac{q-2}{2}} \varphi \bar{L}_{q} u\right\|_{2 \frac{k-1}{q}}^{2} \\
+c_{5}\left\|\varphi L_{q} \bar{L}_{q} u\right\|_{2 \frac{k-1}{q}-\frac{1}{2}}^{2}+c_{6}\left\|t^{2 k+\frac{q-4}{2}} \varphi u\right\|_{2 \frac{k-1}{q}}^{2}+c_{7}\left\|t^{2 k-1} \varphi L_{q} u\right\|_{2 \frac{k-1}{q}-\frac{1}{2}}^{2} \\
=\sum_{j=0}^{7} c_{j} E_{j} \lesssim\|\varphi \mathcal{P} u\|_{2 \frac{k-1}{q}}^{2}+\text { R.J. }
\end{gathered}
$$

where the $c_{j}$ are suitable constants. 
As before we start by estimating $E_{0}$ :

$$
\|\varphi u\|_{0}^{2}=\left|\left(\left[L_{q}, t\right] \varphi u, \varphi u\right)\right| \leq \underbrace{\left|\left(t L_{q} \varphi u, \varphi u\right)\right|}_{I I}+\underbrace{\left|\left(L_{q} t \varphi u, \varphi u\right)\right|}_{I} .
$$

We consider each term, one by one:

$$
(I) \leq s . c .\|\varphi u\|_{0}^{2}+\left\|\varphi \bar{L}_{q} u\right\|_{0}^{2}+\left\|t^{q} \varphi^{(1)} u\right\|_{0}^{2}+\left\|t^{q} \tilde{\varphi} u\right\|_{-1}^{2}+\text { R.J. },
$$

the last two terms are relative junk in view of (2.7), and

$$
\begin{aligned}
(I I) & \leq\left\|t \varphi L_{q} u\right\|_{0}^{2}+\text { s.c. }\|\varphi u\|_{0}^{2}+\text { R.J. } \\
& \leq\left\|t^{k} \varphi L_{q} u\right\|_{\frac{k-1}{q}}^{2}+\text { s.c. }\left\|\varphi L_{q} u\right\|_{-\frac{1}{q}}^{2}+\text { s.c. }\|\varphi u\|_{0}^{2}+\text { R.J. }
\end{aligned}
$$

Thus we have to estimate the second term of the above inequality. First of all we remark that there exists a symbol $b \in S_{1,0}^{-1}$, unique modulo $S^{-\infty}$, such that $b \sim \sigma([[L, \varphi], \varphi])$, and in view of the estimate (2.7) we have

$$
\begin{aligned}
& \left\|\varphi L_{q} u\right\|_{-\frac{1}{q}}^{2} \leq\left|\left(\bar{L}_{q} \varphi L_{q} u, \varphi u\right)_{-\frac{1}{q}}\right|+\left|\left(\varphi L_{q} u,\left[L_{q}, \varphi\right] u\right)_{-\frac{1}{q}}\right| \\
& \leq\left|\left(\bar{L}_{q} \varphi L_{q} u, \varphi u\right)_{-\frac{1}{q}}\right|+\text { s.c. }\left\|\varphi L_{q} u\right\|_{-\frac{1}{q}}^{2}+\text { R.J. } \\
& \leq\left|\left(\left[\bar{L}_{q} \varphi\right] L_{q} u, \varphi u\right)_{-\frac{1}{q}}\right|+\left|\left(\varphi \bar{L}_{q} L_{q} u, \varphi u\right)_{-\frac{1}{q}}\right|+s . c .\left\|\varphi L_{q} u\right\|_{-\frac{1}{q}}^{2}+R . J . \\
& \lesssim+\left|\left(t^{q-2} \varphi \sqrt{-\Delta_{x}} u, \varphi u\right)_{-\frac{1}{q}}\right|+\left|\left(\varphi L_{q} u,\left[L_{q}, \varphi\right] u\right)_{-\frac{1}{q}}\right|+\text { s.c. }\left\|\varphi L_{q} u\right\|_{-\frac{1}{q}}^{2} \\
& +\left|\left(\varphi L_{q} \bar{L}_{q} u, \varphi u\right)_{-\frac{1}{q}}\right|+\left|\left(L_{q} u,\left[\left[L_{q}, \varphi\right], \varphi\right] u\right)_{-\frac{1}{q}}\right|+R . J . \\
& \lesssim\left\|\varphi \bar{L}_{q} u\right\|_{-\frac{1}{q}}^{2}+\left|\left(\varphi \bar{L}_{q} u,\left[\bar{L}_{q}, \varphi\right] u\right)_{-\frac{1}{q}}\right|+\left|\left(\bar{L}_{q} u,\left[\left[\bar{L}_{q}, \varphi\right], \varphi\right] u\right)_{-\frac{1}{q}}\right| \\
& +\left|\left(\varphi L_{q} u,\left[L_{q}, \varphi\right] u\right)_{-\frac{1}{q}}\right|+\left|\left(t^{q-2}\left[\varphi, \sqrt{-\Delta_{x}}\right] u, \varphi u\right)_{-\frac{1}{q}}\right| \\
& +\left|\left(L_{q} u,\left[\left[L_{q}, \varphi\right], \varphi\right] u\right)_{-\frac{1}{q}}\right|+\left\|t^{\frac{q-2}{2}} \varphi u\right\|_{\frac{1}{2}-\frac{1}{q}}^{2}+\text { s.c. }\left\|\varphi L_{q} u\right\|_{-\frac{1}{q}}^{2}+\text { R.J. } \\
& \lesssim\left\|\varphi \bar{L}_{q} u\right\|_{-\frac{1}{q}}^{2}+\left\|t^{q-1} \varphi^{(1)} u\right\|_{-\frac{1}{q}}^{2}+\left\|\tilde{\varphi} \bar{L}_{q} u\right\|_{-\frac{1}{2}-\frac{1}{q}}^{2}+\left\|t^{\frac{q-2}{2}} \varphi u\right\|_{\frac{1}{2}-\frac{1}{q}}^{2} \\
& +\left\|t^{q-2} \varphi^{(1)} u\right\|_{-\frac{1}{q}}^{2}+\|\varphi u\|_{-\frac{1}{q}}^{2}+\left\|\tilde{\varphi} \bar{L}_{q} u\right\|_{-\frac{1}{2}-\frac{1}{q}}^{2}+\text { s.c. }\left\|\varphi L_{q} u\right\|_{-\frac{1}{q}}^{2}+\text { R.J. } \\
& \lesssim\left\|t^{\frac{q-2}{2}} \varphi u\right\|_{\frac{1}{2}-\frac{1}{q}}^{2}+s . c .\left\|\varphi L_{q} u\right\|_{-\frac{1}{q}}^{2}+R . J .
\end{aligned}
$$

Now using (2.1) and (2.3) we have

$$
\begin{aligned}
\left\|t^{\frac{q-2}{2}} \varphi u\right\|_{\frac{1}{2}-\frac{1}{q}}^{2} \leq & \text { l.c. }\left\|t^{\frac{q-2}{2}} t^{k} \varphi u\right\|_{\frac{1}{2}+\frac{k-1}{q}}^{2}+\text { s.c. }\|\varphi u\|_{0}^{2} \\
\lesssim & \text { l.c. }\left(\left\|L_{q} t^{k} \varphi u\right\|_{\frac{k-1}{q}}^{2}+\left\|t^{k} \varphi u\right\|_{\frac{k-1}{q}}^{2}\right)+\text { s.c. }\|\varphi u\|_{0}^{2} \\
\lesssim & \text { l.c. }\left\|t^{k} \varphi L_{q} u\right\|_{\frac{k-1}{q}}^{2}+\text { l.c. }\left\|t^{k}\left[L_{q}, \varphi\right] u\right\|_{\frac{k-1}{q}}^{2}+\text { l.c. }\left\|t^{k-1} \varphi u\right\|_{\frac{k-1}{q}}^{2} \\
& + \text { l.c. }\left\|t^{k} \varphi u\right\|_{\frac{k-1}{q}}^{2}+\text { s.c. }\|\varphi u\|_{0}^{2} \\
\lesssim & \text { l.c. }\left\|t^{k} \varphi L_{q} u\right\|_{\frac{k-1}{q}}^{2}+\text { l.c. }\left\|t^{k+q-1} \varphi^{(1)} u\right\|_{\frac{k-1}{q}}+\text { l.c. }\left\|t^{k+q-1} \tilde{\varphi} u\right\|_{\frac{k-1}{q}-1} \\
& + \text { l.c. }\left\|t^{k-1} \varphi u\right\|_{\frac{k-1}{q}}^{2}+\text { l.c. }\left\|t^{k} \varphi u\right\|_{\frac{k-1}{q}}^{2}+\text { s.c. }\|\varphi u\|_{0}^{2}+\text { R.J. }
\end{aligned}
$$


The next to last term, i.e., $\left\|t^{k} \varphi u\right\|_{\frac{k-1}{q}}^{2}$, is relative junk because of (2.1), (2.3). The same is true for the term $\left\|t^{k-1} \varphi u\right\|_{\frac{k-1}{q}}^{{ }^{2}}$; indeed using $\left[L_{q}, t^{2 k-1}\right]=(2 k-1) t^{2 k-2}$, we have

$$
\begin{aligned}
\left\|t^{k-1} \varphi u\right\|_{\frac{\frac{k-1}{q}}{q}}^{2} & \lesssim\left\|\varphi \bar{L}_{q} u\right\|_{\frac{k-1}{q}}^{2}+\left\|\varphi t^{k} L_{q} u\right\|_{\frac{k-1}{q}}^{2}+\text { s.c. }\left\|t^{k-1} \varphi u\right\|_{\frac{k-1}{q}}^{2} \\
& +\left\|t^{2 k-1} \varphi u\right\|_{\frac{k-1}{q}}^{2}+\left\|t^{k+q-1} \varphi^{(1)} u\right\|_{\frac{k-1}{q}}^{2}+\left\|t^{k+q-1} \tilde{\varphi} u\right\|_{\frac{k-1}{q}-1}^{2}+R . J .
\end{aligned}
$$

On the other hand we have

$$
\left\|t^{k+q-1} \varphi^{(1)} u\right\|_{\frac{k-1}{q}}^{2} \lesssim \underbrace{\left\|t^{\frac{q}{2}} \varphi^{(1)} u\right\|_{-\frac{1}{2}}^{2}+\left\|t^{2 k+\frac{q-2}{2}-1} u\right\|_{2 \frac{k-1}{q}-\frac{1}{2}}^{2}}_{R . J .},
$$

which are both relative junk by (2.7) and by definition.

We can conclude that

$$
\left\|t^{\frac{q-2}{2}} \varphi u\right\|_{\frac{1}{2}-\frac{1}{q}}^{2} \lesssim\left\|\varphi \bar{L}_{q} u\right\|_{\frac{k-1}{q}}^{2}+\left\|\varphi t^{k} L_{q} u\right\|_{\frac{k-1}{q}}^{2}+\text { s.c. }\|\varphi u\|_{0}^{2}+\text { R.J. }
$$

and then

$$
(I I) \lesssim\left\|\varphi \bar{L}_{q} u\right\|_{\frac{k-1}{q}}^{2}+\left\|\varphi t^{k} L_{q} u\right\|_{\frac{k-1}{q}}^{2}+\text { s.c. }\|\varphi u\|_{0}^{2}+R . J .
$$

Using the above considerations on $(I)$ and $(I I)$ and putting them together, we obtain

$$
\|\varphi u\|_{0}^{2} \lesssim\left\|\varphi \bar{L}_{q} u\right\|_{\frac{k-1}{q}}^{2}+\left\|\varphi t^{k} L_{q} u\right\|_{\frac{k-1}{q}}^{2}+\|\varphi Q u\|_{\frac{k-1}{q}}^{2}+R . J .
$$

We proceed to estimate $E_{2}, E_{3}$ and $E_{4}$ of (2.9):

$$
\begin{aligned}
\left\|\varphi \bar{L}_{q} u\right\|_{\frac{k-1}{q}}^{2}+ & \left\|t^{k} \varphi L_{q} u\right\|_{\frac{k-1}{q}}^{2}+\|\varphi Q u\|_{\frac{k-1}{q}}^{2} \\
\lesssim & l . c .\|\varphi P u\|_{2 \frac{k-1}{q}}^{2}+\text { s.c. }\|\varphi u\|_{0}^{2}+\left|\left(\varphi \bar{L}_{q} u,\left[\bar{L}_{q}, \varphi\right] u\right)_{\frac{k-1}{q}}\right| \\
& +\left|\left(\bar{L}_{q} u,\left[\left[\bar{L}_{q}, \varphi\right], \varphi\right] u\right)_{\frac{k-1}{q}}\right|+\left|\left(\varphi t^{k} L_{q} u, t^{k}\left[L_{q}, \varphi\right] u\right)_{\frac{k-1}{q}}\right| \\
& +\left|\left(t^{2 k} L_{q} u,\left[\left[L_{q}, \varphi\right], \varphi\right] u\right)_{\frac{k-1}{q}}\right| \\
\lesssim & l . c .\|\varphi \mathcal{P} u\|_{2 \frac{k-1}{q}}^{2}+\text { s.c. }\|\varphi u\|_{0}^{2}+\text { s.c. }\left\|t^{\frac{q-2}{2}} \varphi \bar{L}_{q} u\right\|_{2 \frac{k-1}{q}}^{2}+l . c .\left\|t^{\frac{q}{2}} \varphi^{(1)} u\right\|_{0}^{2} \\
& + \text { l.c. }\left\|t^{\frac{q}{2}} \tilde{\varphi} u\right\|_{-1}^{2}+\text { s.c. }\left\|t^{\frac{q-2}{2}} \tilde{\varphi} \bar{L}_{q} u\right\|_{2 \frac{k-1}{q}-\frac{1}{2}}^{2}+l . c .\left\|t^{\frac{q}{2}} \tilde{\varphi} u\right\|_{-\frac{1}{2}}^{2} \\
& + \text { s.c. }\left\|\varphi t^{k} L_{q} u\right\|_{\frac{k-1}{q}}^{2}+\text { l.c. }\left\|t^{k+q-1} \varphi^{(1)} u\right\|_{\frac{k-1}{q}}^{2}+\text { l.c. }\left\|t^{k+q-1} \tilde{\varphi} u\right\|_{\frac{k-1}{q}-1}^{2} \\
& + \text { s.c. }\left\|\tilde{\varphi} t^{k} L_{q} u\right\|_{\frac{k-1}{q}-\frac{1}{2}}^{2}+\text { l.c. }\left\|t^{k+q-1} \tilde{\varphi} u\right\|_{\frac{k-1}{q}-\frac{1}{2}}^{2}+R . J . \\
\lesssim & \text { l.c. }\|\varphi \mathcal{P} u\|_{2 \frac{k-1}{q}}^{2}+\text { s.c. }\|\varphi u\|_{0}^{2}+\text { s.c. }\left\|t^{\frac{q-2}{2}} \varphi \bar{L}_{q} u\right\|_{2 \frac{k-1}{q}}^{2}+R . J . \\
= & \text { l.c. }\|\varphi \mathcal{P} u\|_{2 \frac{k-1}{q}}^{2}+\text { s.c.E } E_{4}+\text { s.c. }\|\varphi u\|_{0}^{2}+R . J .
\end{aligned}
$$

We point out that $E_{4}$ has a small constant as a factor, which allows us to absorb it on the left-hand side of (2.9). 
As for the terms $E_{4}$ and $E_{5}$ of (2.9), using (2.4), we have

$$
\begin{aligned}
\left\|t^{\frac{q-2}{2}} \varphi \bar{L}_{q} u\right\|_{2 \frac{k-1}{q}}^{2} & +\left\|\varphi L_{q} \bar{L}_{q} u\right\|_{2 \frac{k-1}{q}-\frac{1}{2}}^{2} \\
& \lesssim\left\|L_{q} \varphi \bar{L}_{q}\right\|_{2 \frac{k-1}{q}-\frac{1}{2}}^{2}+\left\|\bar{L}_{q} \varphi \bar{L}_{q}\right\|_{2 \frac{k-1}{q}-\frac{1}{2}}^{2}+\left\|\varphi L_{q} \bar{L}_{q} u\right\|_{2 \frac{k-1}{q}-\frac{1}{2}}^{2}+R . J . \\
& \lesssim\left\|\varphi L_{q} \bar{L}_{q} u\right\|_{2 \frac{k-1}{q}-\frac{1}{2}}^{2}+\text { R.J. }
\end{aligned}
$$

Thus we consider the only significant term in the last line. To deal with it we split it into three parts:

$$
\begin{aligned}
\left\|\varphi L_{q} \bar{L}_{q} u\right\|_{2 \frac{k-1}{q}-\frac{1}{2}}^{2}= & \left(\varphi L_{q} \bar{L}_{q} u, \varphi L_{q} \bar{L}_{q} u\right)_{2 \frac{k-1}{q}-\frac{1}{2}} \\
= & \left(\varphi \bar{L}_{q} L_{q} \bar{L}_{q} u, \varphi \bar{L}_{q} u\right)_{2 \frac{k-1}{q}-\frac{1}{2}}+\left(\varphi L_{q} \bar{L}_{q} u,\left[\varphi, L_{q}\right] \bar{L}_{q} u\right)_{2 \frac{k-1}{q}-\frac{1}{2}} \\
& \quad+\left(\left[\bar{L}_{q}, \varphi\right] L_{q} \bar{L}_{q} u, \varphi \bar{L}_{q} u\right)_{2 \frac{k-1}{q}-\frac{1}{2}} \\
= & I_{1}+I_{2}+I_{3} .
\end{aligned}
$$

We estimate each one of the above terms.

For $I_{1}$ we have

$$
\begin{aligned}
I_{1} \lesssim & I_{1}+\left\|\varphi \bar{L}_{q} Q u\right\|_{2 \frac{k-1}{q}-\frac{1}{2}}^{2} \\
\lesssim & \mid\left(\varphi \bar{L}_{q} L_{q} \bar{L}_{q} u, \varphi \bar{L}_{q} u\right)_{2 \frac{k-1}{q}-\frac{1}{2}}+\left(\varphi \bar{L}_{q} \bar{L}_{q} t^{2 k} L_{q} u, \varphi \bar{L}_{q} u\right)_{2 \frac{k-1}{q}-\frac{1}{2}} \\
& +\left(\varphi \bar{L}_{q} Q^{2} u, \varphi \bar{L}_{q} u\right)_{2 \frac{k-1}{q}-\frac{1}{2}} \mid+\underbrace{\left|\left(\varphi \bar{L}_{q} \bar{L}_{q} t^{2 k} L_{q} u, \varphi \bar{L}_{q} u\right)_{2 \frac{k-1}{q}-\frac{1}{2}}\right|}_{I_{4}} \\
= & \left|\left(\varphi \bar{L}_{q} \mathcal{P} u, \varphi \bar{L}_{q} u\right)_{2 \frac{k-1}{q}-\frac{1}{2}}\right|+I_{4} \\
\lesssim & \left|\left(\varphi \mathcal{P} u, \varphi L_{q} \bar{L}_{q} u\right)_{2 \frac{k-1}{q}-\frac{1}{2}}\right|+2\left|\left(\varphi \mathcal{P} u,\left[L_{q}, \varphi\right] \bar{L}_{q} u\right)_{2 \frac{k-1}{q}-\frac{1}{2}}\right| \\
& +\left|\left(\mathcal{P} u,\left[\left[L_{q}, \varphi\right], \varphi\right] \bar{L}_{q} u\right)_{2 \frac{k-1}{q}-\frac{1}{2}}\right|+I_{4} \\
\lesssim & l . c .\|\varphi \mathcal{P} u\|_{2 \frac{k-1}{q}-\frac{1}{2}}^{2}+\text { s.c. }\left\|\varphi L_{q} \bar{L}_{q} u\right\|_{2 \frac{k-1}{q}-\frac{1}{2}}^{2}+\|\tilde{\varphi} \mathcal{P} u\|_{2 \frac{k-1}{q}-\frac{1}{2}}^{2} \\
& +\left\|t^{\frac{q-2}{2}} \tilde{\varphi} \bar{L}_{q} u\right\|_{2 \frac{k-1}{q}-\frac{3}{2}}^{2}+\left\|t^{\frac{q-2}{2}} \varphi^{(1)} \bar{L}_{q} u\right\|_{2 \frac{k-1}{q}-\frac{1}{2}}^{2}+I_{4}+R . J . \\
= & \|\varphi \mathcal{P} u\|_{2 \frac{k-1}{q}-\frac{1}{2}}^{2}+I_{4}+\text { s.c.E } E_{5}+R . J . ;
\end{aligned}
$$

for $I_{2}$ we have

$$
\begin{aligned}
\left|I_{2}\right| & =\left|\left(\varphi L_{q} \bar{L}_{q} u,\left[\varphi, L_{q}\right] \bar{L}_{q} u\right)_{2 \frac{k-1}{q}-\frac{1}{2}}\right| \\
& \lesssim s . c .\left\|\varphi L_{q} \bar{L}_{q} u\right\|_{2 \frac{k-1}{q}-\frac{1}{2}}^{2}+\left\|t^{\frac{q-2}{2}} \varphi^{(1)} \bar{L}_{q} u\right\|_{2 \frac{k-1}{q}-\frac{1}{2}}^{2}+R . J . \\
& =\text { s.c. } E_{5}+R . J . ;
\end{aligned}
$$

and for $I_{3}$ we have

$$
\begin{aligned}
\left|I_{3}\right| & =\left|\left(\left[\bar{L}_{q}, \varphi\right] L_{q} \bar{L}_{q} u, \varphi \bar{L}_{q} u\right)_{2 \frac{k-1}{q}-\frac{1}{2}}\right| \\
& \leq\left|\left(\varphi L_{q} \bar{L}_{q} u,\left[\bar{L}_{q}, \varphi\right] \bar{L}_{q} u\right)_{2 \frac{k-1}{q}-\frac{1}{2}}\right|+\left|\left(L_{q} \bar{L}_{q} u,\left[\left[\bar{L}_{q}, \varphi\right], \varphi\right] \bar{L}_{q} u\right)_{2 \frac{k-1}{q}-\frac{1}{2}}\right| \\
& \lesssim s . c .\left\|\varphi L_{q} \bar{L}_{q} u\right\|_{2 \frac{k-1}{q}-\frac{1}{2}}^{2}+\left\|t^{\frac{q-2}{2}} \varphi^{(1)} \bar{L}_{q} u\right\|_{2 \frac{k-1}{q}-\frac{1}{2}}^{2}+\left\|\tilde{\varphi} L_{q} \bar{L}_{q} u\right\|_{2 \frac{k-1}{q}-1}^{2}+\text { R.J. } \\
& =\text { s.c.E } E_{5}+\text { R.J. }
\end{aligned}
$$


Let us now consider $I_{4}$ on the right of the estimate for $I_{1}$ :

$$
I_{4} \leq\left|\left(\varphi \bar{L}_{q} t^{2 k} \bar{L}_{q} L_{q} u, \varphi \bar{L}_{q} u\right)_{2 \frac{k-1}{q}-\frac{1}{2}}\right|+\left|\left(\varphi \bar{L}_{q} t^{2 k-1} L_{q} u, \varphi \bar{L}_{q} u\right)_{2 \frac{k-1}{q}-\frac{1}{2}}\right|=\tilde{I}_{5}+\tilde{I}_{6} .
$$

Taking $\tilde{I}_{5}$ into account, we have

$$
\begin{aligned}
\tilde{I}_{5} & \leq\left|\left(\varphi \bar{L}_{q} t^{2 k} L_{q} \bar{L}_{q} u, \varphi \bar{L}_{q} u\right)_{2 \frac{k-1}{q}-\frac{1}{2}}\right|+\left|\left(\varphi \bar{L}_{q} t^{2 k+q-2} \sqrt{-\Delta_{x}} u, \varphi \bar{L}_{q} u\right)_{2 \frac{k-1}{q}-\frac{1}{2}}\right| \\
& =\tilde{I}_{7}+\tilde{I}_{8} .
\end{aligned}
$$

For $\tilde{I}_{7}$ we have

$$
\begin{aligned}
\tilde{I}_{7} \leq & \left|\left(\varphi t^{2 k} L_{q} \bar{L}_{q} u, L_{q} \varphi \bar{L}_{q} u\right)_{2 \frac{k-1}{q}-\frac{1}{2}}\right|+\left|\left(\left[\varphi, \bar{L}_{q}\right] t^{2 k} L_{q} \bar{L}_{q} u, \varphi \bar{L}_{q} u\right)_{2 \frac{k-1}{q}-\frac{1}{2}}\right| \\
\leq & \left|\left(\varphi t^{2 k} L_{q} \bar{L}_{q} u, L_{q} \varphi \bar{L}_{q} u\right)_{2 \frac{k-1}{q}-\frac{1}{2}}\right|+\left|\left(\varphi t^{2 k} L_{q} \bar{L}_{q} u,\left[L_{q}, \varphi\right] \bar{L}_{q} u\right)_{2 \frac{k-1}{q}-\frac{1}{2}}\right| \\
& +\left|\left(t^{2 k} L_{q} \bar{L}_{q} u,\left[\left[L_{q}, \varphi\right], \varphi\right] \bar{L}_{q} u\right)_{2 \frac{k-1}{q}-\frac{1}{2}}\right| \\
\leq & \left|\left(\varphi t^{2 k} L_{q} \bar{L}_{q} u, \varphi L_{q} \bar{L}_{q} u\right)_{2 \frac{k-1}{q}-\frac{1}{2}}\right|+2\left|\left(\varphi t^{2 k} L_{q} \bar{L}_{q} u,\left[L_{q}, \varphi\right] \bar{L}_{q} u\right)_{2 \frac{k-1}{q}-\frac{1}{2}}\right| \\
& +\left|\left(t^{2 k} L_{q} \bar{L}_{q} u,\left[\left[L_{q}, \varphi\right], \varphi\right] \bar{L}_{q} u\right)_{2 \frac{k-1}{q}-\frac{1}{2}}\right| \\
\lesssim & \text { s.c. }\left\|\varphi L_{q} \bar{L}_{q} u\right\|_{2 \frac{k-1}{q}-\frac{1}{2}}+\text { l.c. }\left\|t^{\frac{q-2}{2}} \varphi^{(1)} \bar{L}_{q} u\right\|_{2 \frac{k-1}{q}-\frac{1}{2}}^{2}+\left\|t^{\frac{q-2}{2}} \tilde{\varphi} \bar{L}_{q} u\right\|_{2 \frac{k-1}{q}-1}^{2} \\
& \quad+\left\|\tilde{\varphi} L_{q} \bar{L}_{q} u\right\|_{2 \frac{k-1}{q}-1}+\text { R.J. } \\
\lesssim & \text { s.c.E } E_{5}+\text { R.J., }
\end{aligned}
$$

while for $\tilde{I}_{8}$ we have

$$
\begin{aligned}
\tilde{I}_{8} \leq & \left|\left(\varphi \bar{L}_{q} t^{2 k+q-2} u, \varphi \bar{L}_{q} u\right)_{2 \frac{k-1}{q}}\right|+\left|\left(\left[\varphi, \sqrt{-\Delta_{x}}\right] \bar{L}_{q} t^{2 k+q-2} u, \varphi \bar{L}_{q} u\right)_{2 \frac{k-1}{q}-\frac{1}{2}}\right| \\
\lesssim & \left|\left(\varphi t^{2 k+q-2} \bar{L}_{q} u, \varphi \bar{L}_{q} u\right)_{2 \frac{k-1}{q}}\right|+\left|\left(\bar{L}_{q} t^{2 k+q-2} u,\left[\left[\sqrt{-\Delta_{x}}, \varphi\right], \varphi\right] \bar{L}_{q} u\right)_{2 \frac{k-1}{q}-\frac{1}{2}}\right| \\
& +\left|\left(\varphi \bar{L}_{q} t^{2 k+q-2} u,\left[\varphi, \sqrt{-\Delta_{x}}\right] \bar{L}_{q} u\right)_{2 \frac{k-1}{q}-\frac{1}{2}}\right|+\left|\left(\varphi t^{2 k+q-3} u, \varphi \bar{L}_{q} u\right)_{2 \frac{k-1}{q}}\right| \\
\lesssim & \left|\left(\varphi t^{2 k+q-3} u,\left[\varphi, \sqrt{-\Delta_{x}}\right] \bar{L}_{q} u\right)_{2 \frac{k-1}{q}-\frac{1}{2}}\right|+l . c .\left\|\varphi t^{2 k+\frac{q-4}{2}} u\right\|_{2 \frac{k-1}{q}}^{2} \\
& +\left|\left(\varphi t^{2 k+q-2} \bar{L}_{q} u,\left[\varphi, \sqrt{-\Delta_{x}}\right] \bar{L}_{q} u\right)_{2 \frac{k-1}{q}-\frac{1}{2}}\right|+s . c .\left\|t^{\frac{q-2}{2}} \varphi \bar{L}_{q} u\right\|_{2 \frac{k-1}{q}}^{2} \\
& +\left|\left(t^{2 k+q-3} u,\left[\left[\sqrt{-\Delta_{x}}, \varphi\right], \varphi\right] \bar{L}_{q} u\right)_{2 \frac{k-1}{q}-\frac{1}{2}}\right| \\
& +\left|\left(t^{2 k+q-2} \bar{L}_{q} u,\left[\left[\sqrt{-\Delta_{x}}, \varphi\right], \varphi\right] \bar{L}_{q} u\right)_{2 \frac{k-1}{q}-\frac{1}{2}}\right| \\
\lesssim & l . c .\left\|\varphi t^{2 k+\frac{q-4}{2}} u\right\|_{2 \frac{k-1}{q}}^{2}+\text { s.c. }\left\|t^{\frac{q-2}{2}} \varphi \bar{L}_{q} u\right\|_{2 \frac{k-1}{q}}^{2}+\left\|t^{\frac{q-2}{2}} \varphi^{(1)} \bar{L}_{q} u\right\|_{2 \frac{k-1}{q}-\frac{1}{2}}^{2} \\
& +\left\|\tilde{\varphi} t^{2 k+\frac{q-4}{2}} u\right\|_{2 \frac{k-1}{q}-1}^{2}+\left\|t^{\frac{q-2}{2}} \tilde{\varphi} \bar{L}_{q} u\right\|_{2 \frac{k-1}{q}-\frac{3}{2}}^{2}+R . J . \\
\lesssim & \text { s.c.E } E_{4}+\text { l.c. } E_{6}+R . J .
\end{aligned}
$$

Hence we get the following estimate for $\tilde{I}_{5}$ :

$$
\tilde{I}_{5} \lesssim \text { s.c. } E_{5}+\text { s.c. } E_{4}+\text { l.c. } E_{6}+\text { R.J. }
$$


To complete the estimate of $I_{4}$, we have to estimate $\tilde{I}_{6}$ :

$$
\begin{aligned}
\tilde{I}_{6} \lesssim & \left|\left(\varphi t^{2 k-1} L_{q} u, L_{q} \varphi \bar{L}_{q} u\right)_{2 \frac{k-1}{q}-\frac{1}{2}}\right|+\left|\left(\left[\varphi, \bar{L}_{q}\right] t^{2 k-1} L_{q} u, \varphi \bar{L}_{q} u\right)_{2 \frac{k-1}{q}-\frac{1}{2}}\right| \\
\lesssim & \left|\left(\varphi t^{2 k-1} L_{q} u, \varphi L_{q} \bar{L}_{q} u\right)_{2 \frac{k-1}{q}-\frac{1}{2}}\right|+2\left|\left(\varphi t^{2 k-1} L_{q} u,\left[L_{q}, \varphi\right] \bar{L}_{q} u\right)_{2 \frac{k-1}{q}-\frac{1}{2}}\right| \\
& +\left|\left(t^{2 k-1} L_{q} u,\left[\left[L_{q}, \varphi\right], \varphi\right] \bar{L}_{q} u\right)_{2 \frac{k-1}{q}-\frac{1}{2}}\right| \\
\lesssim & \left\|\varphi t^{2 k-1} L_{q} u\right\|_{2 \frac{k-1}{q}-\frac{1}{2}}^{2}+\text { s.c. }\left\|L_{q} \varphi \bar{L}_{q} u\right\|_{2 \frac{k-1}{q}-\frac{1}{2}}^{2}+\left\|t^{\frac{q-2}{2}} \varphi^{(1)} \bar{L}_{q} u\right\|_{2 \frac{k-1}{q}-\frac{1}{2}}^{2} \\
& \quad+\left\|\tilde{\varphi} t^{2 k-1} L_{q} u\right\|_{2 \frac{k-1}{q}-1}^{2}+\left\|t^{\frac{q-2}{2}} \tilde{\varphi} \bar{L}_{q} u\right\|_{2 \frac{k-1}{q}-1}^{2}+\text { R.J. } \\
& \lesssim \text { s.c. } E_{4}+\text { s.c. } E_{5}+\text { l.c. } E_{7}+\text { R.J. }
\end{aligned}
$$

We eventually may conclude that

$$
I_{4} \leq \text { s.c. } E_{5}+\text { s.c. } E_{4}+\text { l.c. } E_{6}+\text { l.c. } E_{7}+\text { R.J. }
$$

Summing up, all that we have done can be written as

$$
E_{4}+E_{5} \lesssim\|\varphi \mathcal{P} u\|_{2 \frac{k-1}{q}}^{2}+\text { l.c. }\left(E_{6}+E_{7}\right)+\text { s.c. }\left(E_{4}+E_{5}\right)+\text { R.J. }
$$

We proceed to estimate the last two terms, $E_{6}$ and $E_{7}$ :

$$
\begin{aligned}
\left\|t^{2 k+\frac{q-4}{2}} \varphi u\right\|_{2 \frac{k-1}{q}} & +\left\|t^{2 k-1} \varphi L_{q} u\right\|_{2 \frac{k-1}{q}-\frac{1}{2}}^{2} \\
\lesssim & \left\|t^{2 k-1} \varphi L_{q} u\right\|_{2 \frac{k-1}{q}-\frac{1}{2}}^{2}+\left\|t^{2 k-1} \varphi u\right\|_{2 \frac{k-1}{q}-\frac{1}{2}}^{2}+\left\|t^{2 k-2} \varphi u\right\|_{2 \frac{k-1}{q}-\frac{1}{2}}^{2} \\
& +\left\|t^{2 k+q-2} \varphi_{1} u\right\|_{2 \frac{k-1}{q}-\frac{1}{2}}^{2}+\left\|t^{2 k+q-2} \tilde{\varphi} u\right\|_{2 \frac{k-1}{q}-\frac{3}{2}}^{2}+R . J .
\end{aligned}
$$

Except for the first term, the other terms are all good relative junk. Hence we have

$$
\begin{aligned}
\| t^{2 k-1} & \varphi L_{q} u \|_{2 \frac{k-1}{q}-\frac{1}{2}}^{2} \\
\leq & \left\|t^{2 k-1} \varphi L_{q} u\right\|_{2 \frac{k-1}{q}-\frac{1}{2}}^{2}+\left\|t^{2 k-1} \varphi Q u\right\|_{2 \frac{k-1}{q}-\frac{1}{2}}^{2} \\
\leq & \mid\left(\varphi L_{q} \bar{L}_{q} u, t^{2 k-2} \varphi u\right)_{2 \frac{k-1}{q}-\frac{1}{2}}+\left(\varphi \bar{L}_{q} t^{2 k} L_{q} u, t^{2 k-2} \varphi u\right)_{2 \frac{k-1}{q}-\frac{1}{2}} \\
& +\left(\varphi Q^{2} u, t^{2 k-2} \varphi u\right)_{2 \frac{k-1}{q}-\frac{1}{2}}|+|\left(\varphi L_{q} \bar{L}_{q} u, t^{2 k-2} \varphi u\right)_{2 \frac{k-1}{q}-\frac{1}{2}} \mid \\
& +\mid \underbrace{\left|\left(\left[\bar{L}_{q}, t^{2 k-2} \varphi^{2}\right] t^{2 k} L_{q} u, u\right)_{2 \frac{k-1}{q}-\frac{1}{2}}\right|}_{=I_{9}} \\
\lesssim & \|\varphi \mathcal{P} u\|_{2 \frac{k-1}{q}-\frac{1}{2}}^{2}+\text { l.c. }\left\|t^{2 k-2} \varphi u\right\|_{2 \frac{k-1}{q}-\frac{1}{2}}^{2}+\text { s.c. }\left\|\varphi L \bar{L}_{q} u\right\|_{2 \frac{k-1}{q}-\frac{1}{2}}^{2}+I_{9} \\
= & \|\varphi \mathcal{P} u\|_{2 \frac{k-1}{q}-\frac{1}{2}}^{2}+\text { s.c. } E_{5}+I_{9}+\text { R.J. }
\end{aligned}
$$

We have only to estimate $I_{9}$ :

$$
\begin{aligned}
I_{9} \lesssim \text { s.c. }\left\|t^{2 k-1} \varphi L_{q} u\right\|_{2 \frac{k-1}{q}-\frac{1}{2}}^{2}+l . c .\left\|t^{2 k-2} \varphi u\right\|_{2 \frac{k-1}{q}-\frac{1}{2}}^{2}+\left\|t^{2 k+q-2} \varphi^{(1)} u\right\|_{2 \frac{k-1}{q}-\frac{1}{2}}^{2} \\
\quad+\left\|t^{2 k+q-2} \tilde{\varphi} u\right\|_{2 \frac{k-1}{q}-\frac{3}{2}}^{2}+\left\|t^{2 k-1} \tilde{\varphi} L_{q} u\right\|_{2 \frac{k-1}{q}-1}^{2}+\left\|t^{2 k+q-2} \tilde{\varphi} u\right\|_{2 \frac{k-1}{q}-1}^{2}+R . J . \\
=\text { s.c. } E_{7}+R . J .
\end{aligned}
$$

Again, summarizing, we may write that

$$
E_{6}+E_{7} \lesssim\|\varphi \mathcal{P} u\|_{2 \frac{k-1}{q}}^{2}+\text { s.c. }\left(E_{7}+E_{5}\right)+\text { R.J. }
$$


The joint action of this estimate and the estimate (2.10) allows us to obtain the estimate (2.9); hence we have

$$
\|\varphi u\|_{0}^{2} \lesssim\|\tilde{\varphi} \mathcal{P} u\|_{2 \frac{k-1}{q}}^{2}+R . J .
$$

Using the previous techniques, we can push down arbitrarily the Sobolev index of the R.J. and for some $\tilde{\varphi}$, radial and $\tilde{\varphi} \equiv 1$ near the support of $\varphi$, we have

$$
\|\varphi u\|_{0}^{2} \lesssim\|\tilde{\varphi} \mathcal{P} u\|_{2 \frac{k-1}{q}}^{2}+\|\tilde{\varphi} u\|_{-N}^{2} .
$$

More generally, starting with a generic Sobolev index $s$ in the left-hand side of the estimate (2.9), we can conclude that

$$
\|\varphi u\|_{s}^{2} \lesssim\|\tilde{\varphi} \mathcal{P} u\|_{s+2 \frac{k-1}{q}}^{2}+\|u\|_{-N}^{2}
$$

where again $\tilde{\varphi}$ is radial and equal to 1 on the support of $\varphi$.

Proof of the theorem. To obtain the result, we use the classical convolution argument as in [8], [7] and [2]. We introduce $\chi \in C_{0}^{\infty}$, radial and such that $\chi(0)=1$, and the related smoothing operator $S_{\delta}$ defined by $\widehat{S_{\delta} u}=\chi(\delta\|\xi\|) \widehat{u}(\xi)$ and such that $S_{\delta} u$ converges towards $u$ for $\delta \rightarrow 0$. Replacing $u$ by $S_{\delta} u$ in the above estimate, we obtain that if $P u \in H_{l o c}^{t}(U), U$ an open neighborhood of $(0,0,0)$ and $t<-N$, then there exists $V$, a circular neighborhood of $(0,0,0)$ with $V \subset U$, such that $u \in H_{l o c}^{t-2 \frac{k-1}{q}}(V)$. Arguing by induction on $t$ gives the result.

\section{REFERENCES}

[1] A. Bove, D. S. Tartakoff, Analytic hypoellipticity at non-symplectic Poisson-Treves strata for certain sums of squares of vector fields, J. Geom. Anal. 18 (2008), no. 4, 1002-1021. MR2438908 (2009f:35039)

[2] A. Bove, M. Derridj, J. J. Kohn, D. S. Tartakoff, Sums of squares of complex vector fields and (analytic-)hypoellipticity, Math. Res. Lett. 13 (2006), no. 5-6, 683-701. MR 2280767 (2007k:35051)

[3] A. Bove, M. Derridj, D. S. Tartakoff, Analytic hypoellipticity in the presence of nonsymplectic characteristic points, J. Funct. Anal. 234 (2006), no. 2, 464-472. MR2216906 (2006k:35034)

[4] A. Bove, M. Mughetti, D. S. Tartakoff, Hypoellipticity and nonhypoellipticity for sums of squares of complex vector fields, preprint, 2011.

[5] M. Christ, A remark on sums of squares of complex vector fields, arXiv:math.CV/0503506.

[6] N. Hanges, Analytic regularity for an operator with Treves curves, J. Funct. Anal. 210 (2004), no. 2, 295-320. MR2053489 (2005b:35032)

[7] J. J. Kohn, Hypoellipticity and loss of derivatives. With an appendix by Makhlouf Derridj and David S.Tartakoff, Ann. of Math. (2) 162 (2005), 943-986. MR.2183286 (2006k:35036)

[8] J. J. Kohn, Pseudo-differential operators and hypoellipticity. Partial differential equations (Proc. Sympos. Pure Math., Vol. XXIII, Univ. California, Berkeley, Calif., 1971), pp. 61-69. Amer. Math. Soc., Providence, R.I., 1973. MR0338592 (49:3356)

[9] D. S. Tartakoff, Analyticity for singular sums of squares of degenerate vector fields, Proc. Amer. Math. Soc. 134 (2006), no. 11, 3343-3352. MR.2231919 (2007h:35030)

Dipartimento di Matematica, Università di Bologna, Piazza di Porta S. Donato 5, 40127 Bologna, Italia

E-mail address: chinni@dm.unibo.it 